\title{
BREAKING THE TESTING PYRAMID WITH VIRTUAL TESTING AND HYBRID SIMULATION
}

\author{
Shawn $\mathrm{You}^{1}$, X. Shawn $\mathrm{Gao}^{2}$, Arlin Nelson ${ }^{3}$ \\ ${ }^{1}$ MTS Systems Corporation, 14000 Technology Drive, Eden Prairie, MN 55344, \\ USA; email: shawn.you@mts.com \\ ${ }^{2}$ MTS Systems Corporation, 14000 Technology Drive, Eden Prairie, MN 55344, \\ USA; email: shawn.gao@mts.com \\ ${ }^{3}$ MTS Systems Corporation, 14000 Technology Drive, Eden Prairie, MN 55344, \\ USA; email: arlin.nelson@mts.com
}

\begin{abstract}
Virtual testing and hybrid simulation have become an important trend in airplane design and validation. The traditional Testing Pyramid (or Building Block) approaches that emphasis on uniaxial coupon test and full structure certification test are being challenged. Researchers are trying to use advanced testing and simulation methods to replace the Testing Pyramid approach.

Before physical testing, virtual testing can be conducted to simulate the physical test. Virtual model of the full testing system including controller, actuators, and fixtures can be constructed and validated. In this work, an example has been developed and validated to show the potentials of the virtual testing process.

Hybrid simulation is an approach of analyzing an analysis model and physical structure integrated system under realistic loading conditions. Hybrid simulation combines the lab testing with numerical analysis to explore the benefits of both methodologies. In this study, a hybrid simulation for a simplified airplane wing was conducted to demonstrate the process.

Virtual testing and hybrid simulation are alternative methods of Testing Pyramid approach. Full scale tests are still required for certification but the more that is known about the test article, the greater chances of success in the full-scale certification testing.
\end{abstract}

Keywords: virtual testing, hybrid simulation, reduced order model, cross coupling, ANSYS, Opensees, OpenFresco 


\section{INTRODUCTION}

Each aircraft development effort involves testing and analyzing small samples (coupons) to characterize the material properties and obtain material allowables. After components are available, they will be tested for evaluating strength, fatigue, and failure behavior with multiple combined loading conditions. At the end, the full structure or major subcomponents will be tested in full-scale against predefined certification specifications. Each step along the way is supported by detailed analysis and testing. This development process becomes known as the Testing Pyramid or Building-block approach. Testing Pyramid approach is the backbone of aircraft certification process.

For many years, Testing Pyramid approach has been used to develop many kinds of aircraft. But, it is considered to be expensive and time-consuming especially as increasingly complex composite structures and additive layer manufactured metal materials proliferate in aerospace applications.

Testing Pyramid approach in its current form requires large number of coupon tests to define material properties assuming that these material properties can be applied to large structures. Unfortunately, in many cases, especially dealing with composite materials, coupon level failure data does not correlate well with failure behavior observed on component and substructure levels [1]. To ensure safety, large safety factors have to be applied causing over designed structures.

With increasing pressure of reducing development cost and shortening development cycle, researches are increasingly using advanced modeling tools in combination with physical testing to create better design and make the design evaluation more efficient. Virtual testing and hybrid simulation methods have been created for reducing the time and cost. People call this trend as "Breaking the Pyramid" [1][2].

This work introduces some of the latest advances of virtual testing and hybrid simulation approaches that have been developed and used in aerospace testing.

\section{VIRTUAL TESTING}

Virtual testing is a method that simulates lab testing using analysis software. There are two main types of virtual testing. One type is mainly aimed to predict specimen behaviors, such as stress distribution, resonant frequency, buckling load, and fatigue life, under realistic loading conditions. By doing so, initial designs can be optimized before prototypes are available. After the virtual models are validated by physical tests, they can be used more extensively in the design optimization. There have been many successful virtual testing examples in this aspect [3][4]. The other type of virtual testing is for setting up and conducting physical tests. By rehearsing the physical tests, uncertainties can be understood ahead of time, therefore, ensuring physical testing to be conducted successfully. This work is focused on the latter type of virtual testing.

Due to the increasingly more sophisticated and complex tests that are being conducted worldwide, many of structure tests consist of expensive and fragile test specimens, complicated test setup, advanced control algorithms, and sophisticated error compensation techniques. Complex test specimens have a high level of cross coupling effect among loading actuators. Aero testing can be risky if testing commences without 
previously verifying that all the employed testing components are set up and operating correctly. To ensure safety, stability, and accuracy of the tests, test engineers have to conduct physical tests at a relatively low speed causing extra time to complete the tests. For this reason, an increasing number of test engineers are starting to virtually simulate aero tests prior to their actual execution to make sure that everything will work as expected. In addition to mitigating risk, the virtual simulation of an aero test can also be utilized as a tool to pre-tune control (PID) parameters, which will lead to increased accuracy and confidence during the actual tests. The virtual testing will validate servo valve sizing, hydraulic flow requirements, supply and return accumulation (how much and where) and test profile transition times.

To make the test rehearsal realistic, test specimen, test fixture, hydraulic components, system delay and roll-off, cross-coupling effects, and control and error compensation algorithms need to be modeled in the virtual testing model. There are many methods and software packages that can be used to construct the virtual model. This work used Matlab/Simulink.

Typical aero structural test specimens have load conditions derived from pressure distributions on the surfaces and inertial loads from the payload so the test is run in force control. With loads being applied by actuators, a structural specimen deforms and a dynamic response (displacement, velocity, and acceleration) is being generated. The relationship between the load and the dynamic response can be characterized and mathematical models can be created to mimic the behavior of the test specimen. In addition to the resistance, the energy dissipation and inertia of the specimen can also be modeled. In this way, a virtual specimen is simulated to provide the response for a given command. The virtual specimen can be as simple as a linear-elastic spring or as complicated as a non-linear dynamic finite element model. A sophisticated FEA model can definitely capture the behavior of the specimen accurately. However, a full blown FEA model often requires intensive computation effort. The extra-long solving time can make virtual testing effort impractical. To reduce solving time, a reduced order model method was developed that converts a FEA model into a first order state space model. A state space model can be easily integrated with other dynamical components, such as actuators and controllers, to form a system level of virtual testing model.

State space models are very computational efficient, therefore, it allows real-time execution. Commonly used methods for creating reduced order models include CraigBampton method. This method combines motion of boundary points with modes of the structure assuming the boundary points are held fixed. It accounts for both mass and stiffness. The problem size is defined by frequency range. It allows for different boundary conditions at interface.

Reduced order models are typically used in system level of virtual testing to achieve fast computation speed. The main goal for the system level simulation is to obtain global information, such as forces and displacements at important locations. In this sense, reduced order models do not have big negative impact on model accuracy for many of the aero virtual testing applications because many structures, such as airplane wings, are fairly linear. After solving the system level, the global information obtained from the system level model can be applied to much refined FEA models for specific components to obtain local information, such as local stresses and strains. 
To construct a virtual testing model, hydraulic components need to be modeled. At MTS, Matlab/Simulink models were created for nearly all of hydraulic components, such as actuators, servo valves, accumulators, and hydraulic pumps, MTS manufactures. The actuators models consider effects of volumetric and compressibility flows, crosspiston leakage flow, parasitic damping, additional trapped oil volume, and seal friction were modeled in these actuator blocks. The servo valve models include effects such as bandwidth limitations and flow gain variation due to pressure switching. The accumulator blocks compute hydraulic supply pressure change with net flow demand. These hydraulic component models have been validated and proven to be accurate.

The controller models were built using Simulink as well. The virtual controllers model the closed loop control with all parameters and filters in the physical controllers. The controller models were validated by comparing the output signals of the controller models with the same output signals from the physical controllers for same commands.

The hydraulic component and controller models are modularized so that blocks for different types and sizes of the components can be easily assembled together by changing some parameters. Model parameters are the numeric representations of the physical properties of the physical system that are required as input for the virtual testing models. These parameters are both determined from product literature and from direct measurement of the system.

To improve the solving speed, S-Function (user-defined blocks written in $\mathrm{C}$ and compiled) blocks were used to model calculation intensive components, such as PID controller and matrix transformation.

To demonstrate the process of virtual testing, the test system shown in Figure 1 was used to conduct both physical and virtual tests. This actual test system consists of a realtime controller, four hydraulic actuators, and a steel plate as part of a much simplified airplane wing. Closed loop control was accomplished by a real-time controller that computes servo valve command updates on the basis of force sensor feedbacks. A reduced order model of the plate was created based upon an ANSYS model of that plate. The specimen model was integrated with the hydraulic component and the controller models of this system forming a system level virtual testing model.

To demonstrate the accuracy of this virtual testing system, some square wave command signals were played out through the virtual and physical testing systems. Response signals, such as actuator forces, actuator displacements, and servo valve output, were collected for comparison purpose. Figure 2 shows the comparison of actuator forces from virtual model and physical measurement when P Gain was set to be 6 for Actuator No. 4. The figure shows that the actuator forces matched very well. Also, both virtual and physical testing results showed stable control. Figure 3 shows the actuator force comparison for P Gain to be 8 for the same actuator. The figure still shows good match between model prediction and actual measurement. More importantly, both virtual prediction and physical measurement showed some degree of instability demonstrating the capability of virtual testing in obtaining tuning parameters of the physical system. Figure 4 shows the zoom in view of the square wave response. 


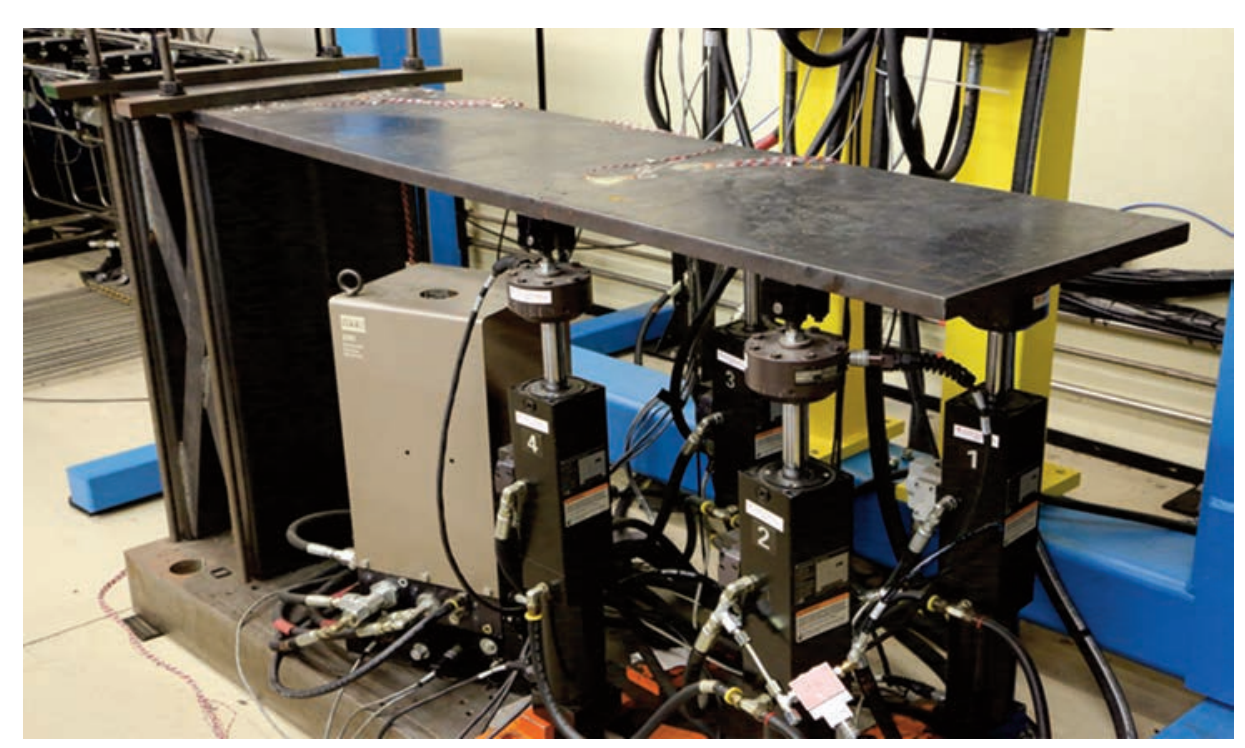

Figure 1. Demonstration test system.

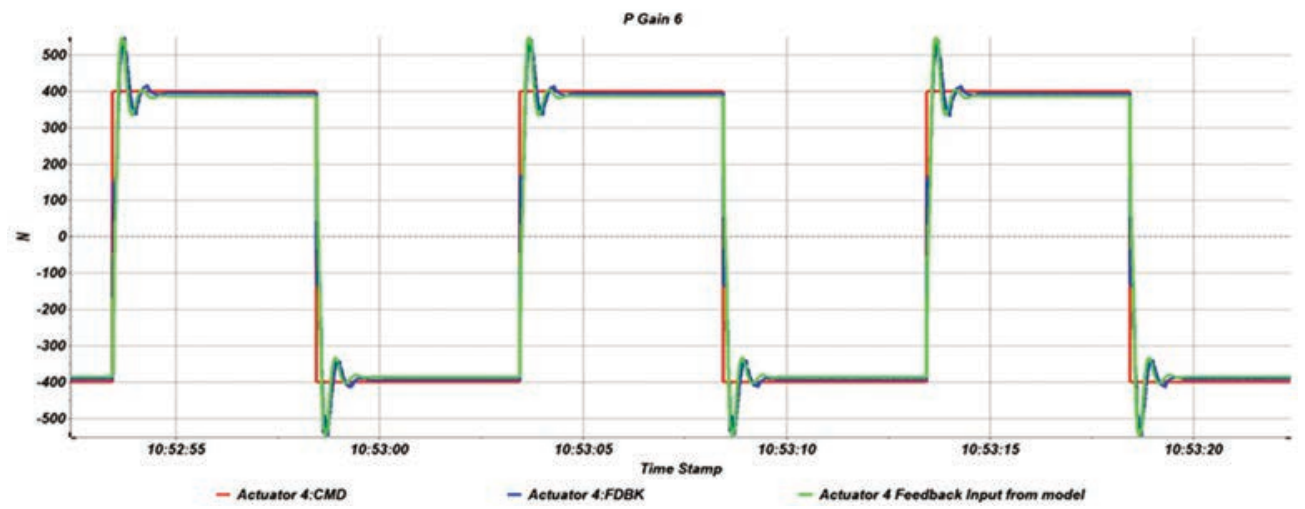

Figure 2. Square wave actuator force response for $\mathbf{P}$ Gain $=6$.

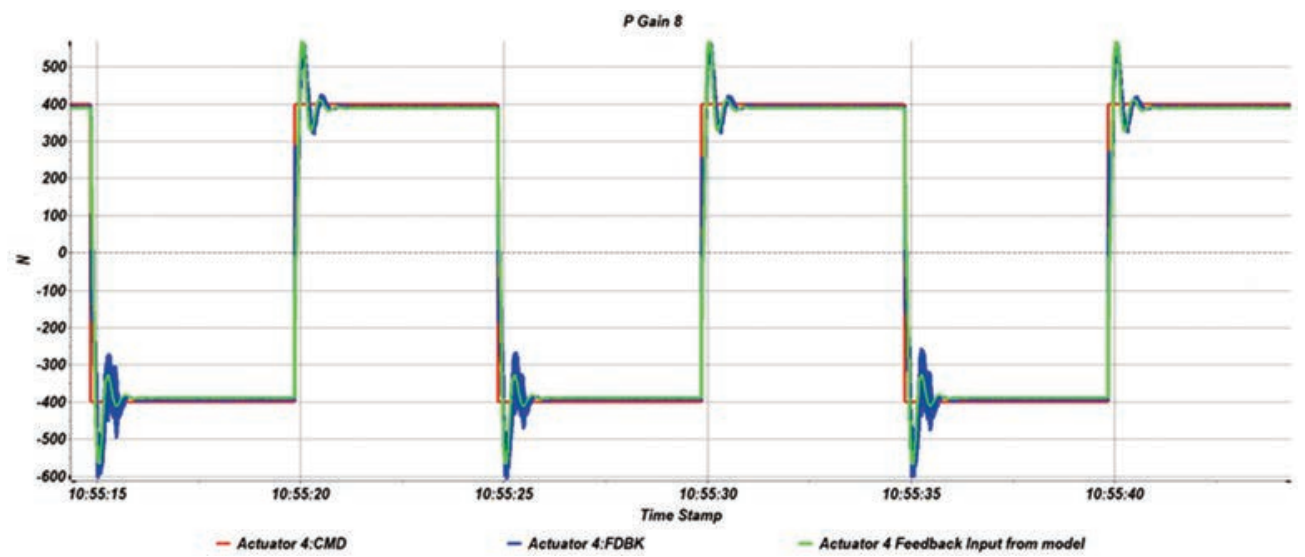

Figure 3. Square wave actuator force response for $\mathbf{P}$ Gain $=8$. 


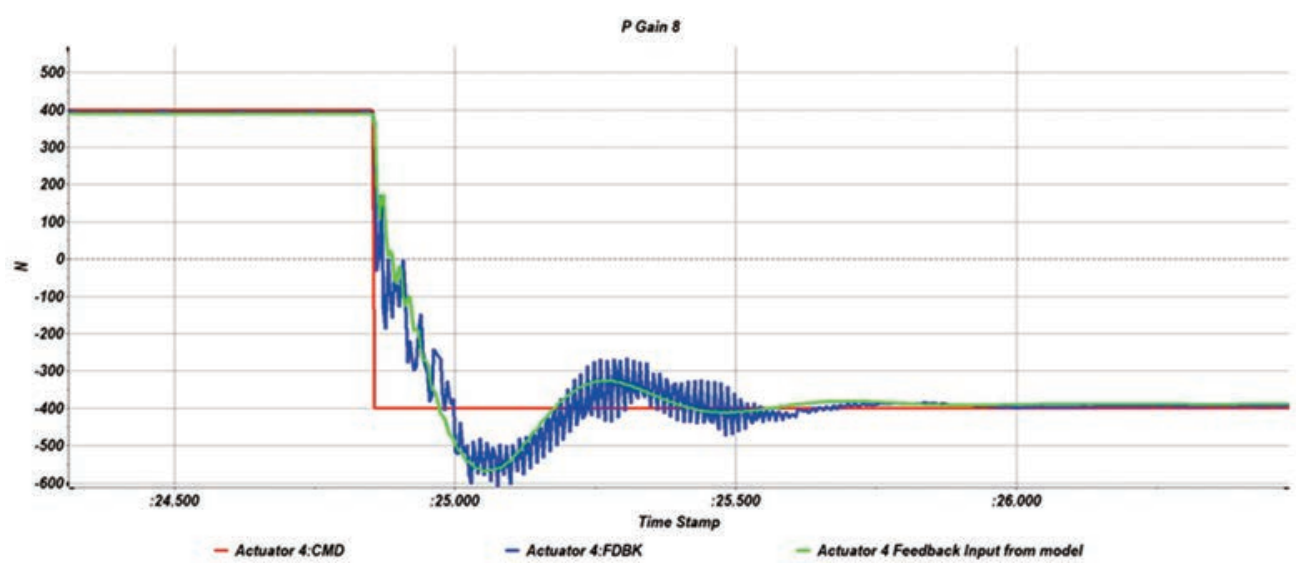

Figure 4. Zoom in square wave response for $\mathbf{P}$ Gain $=8$.

Figure 5 and 6 show actuator displacement and servo valve command comparison between virtual and physical tests. These signals matched very well.

It is worth mentioning that the virtual model was compiled to $\mathrm{C}++$ code and downloaded to a target pc. During test execution, the controller software sent command to both physical controller driving physical actuators and the virtual testing model to conduct virtual testing. The virtual and physical testing were conducted in parallel in real-time demonstrating that the virtual testing model is computationally efficient.

The virtual testing model, with this level of accuracy and efficiency, can be used to accurately mimic the physical tests. Through test rehearsing, test engineers can optimize the test design, discover potential problems, and determine the tuning parameters before physical tests are conducted.
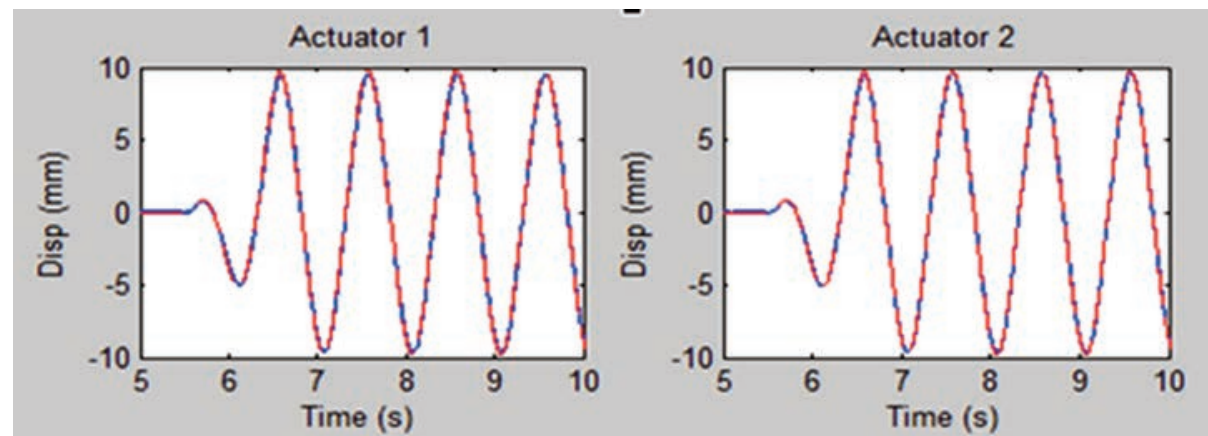

Figure 5. Actuator displacement comparison between virtual and physical tests.
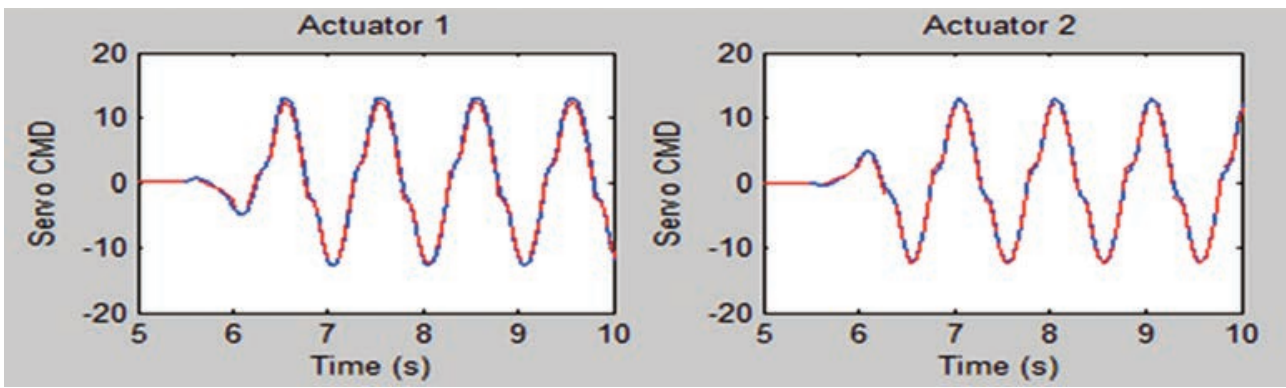

Figure 6. Servo valve CMD comparison between virtual and physical tests. 


\section{HYBRID SIMULATION}

Hybrid simulation is a dynamic experimental testing method where a system is divided into numerically simulated and experimentally tested subassemblies. This method enables the hard to model components to be tested in the lab. As a result, it greatly reduces the requirement for an accurate analysis model and improves the accuracy for the simulation. Hybrid simulation is based on a step-by-step numerical integration of the equations of motion; where, for each analysis step, the numerical model calculates command signals, sends them to the experimental test system, and then collects the resulting response from instrumentation devices.

Researchers had started to conduct hybrid simulation since 1969. Since then, many hybrid simulation methods and software packages have been developed. Hybrid simulation has become a well-accepted testing method in civil, ground vehicle, wind energy, and other industries. Hybrid simulation has been used more and more in aero testing. One example of hybrid simulation in aero testing was the test of a lag damper of a helicopter at University of Bristol [5]. Another example is that MTS is working with an airplane manufacturer in North America to conduct a landing gear hybrid simulation project.

There are different types of hybrid simulation approaches. Real-time hybrid simulation requires both solving of analytical model and loading of physical structure to be real-time. For fast solving, the analysis model needs to be converted into C++ code and run on a pc with a highly efficient operating system. The connection between the analysis model and the test rig controller is mostly through Scramnet interface. Realtime hybrid simulation is required for studying rate dependent specimens.

Another type of hybrid simulation allows analysis model to be solved at a much slower pace. In this case, the physical test only provides response of a structure due to stiffness change. This approach is widely used in studying rate independent structures. In this approach, different analysis packages, such as Ansys, Abaqus, ADAMS, LS DANA, Matlab, Simulink, and Opensees, can be used for model creation and solving. There are different kinds of interfacing software connecting the analysis model and the controller. OpenFresco is one of them. Developed by UC Berkeley, OpenFresco is an open source software for hybrid simulation deployment [8][9]. It uses a user defined experimental element to send displacement commands from FEA models and receive force feedbacks from test systems. MTS has developed an interfacing software (named CSI) that can receive and send signals from and to OpenFresco and map them to different channels in MTS controllers.

To illustrate hybrid simulation process, a quasi-static hybrid test was setup as shown in Figure 7 where a flat plate was divided into two parts. The left part was tested using the test system shown in Figure 1 while the right part of the plate was modeled by Opensees. 


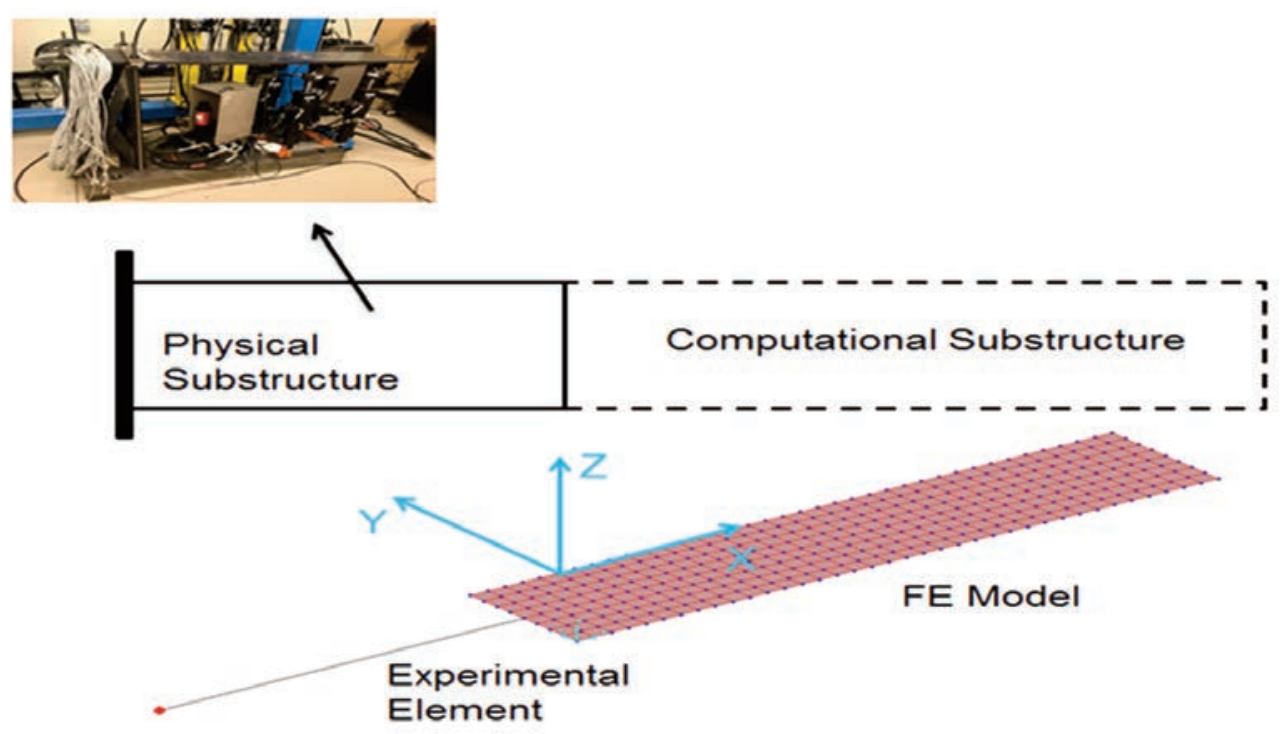

Figure 7. A quasi-static hybrid simulation system testing a flat plate.

Figure 8 shows the data flow of this hybrid test. An experimental element inside the Opensees model communicates with the controller software through OpenFresco and MTS CSI software. For a cyclic loading case, the hybrid test was carried through. Figure 9 shows three commands (two rotational angles and one vertical displacement) and corresponding feedbacks during this event.

This example demonstrates the possibility of testing only a part of an airplane wing while the rest is modeled. This hybrid simulation approach can potentially save significant amount of money and time.

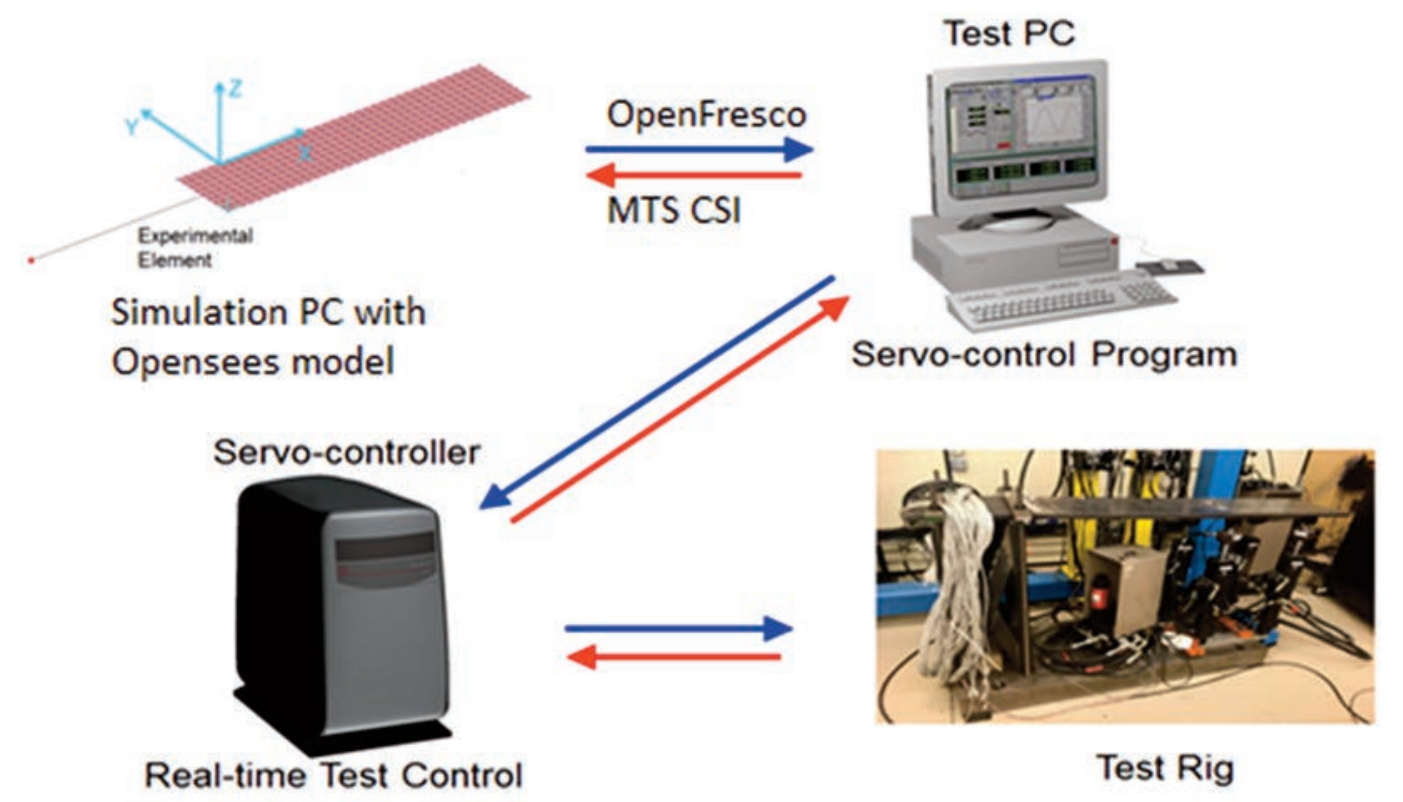

Figure 8. Data flow of the quasi-static hybrid simulation. 




Figure 9. Commands and feedbacks for a sinusoid excitation.

Hybrid simulation offers many possibilities over traditional Testing Pyramid approach. It could potentially increase testing efficiency, reduce cost, and enable tests to be conducted in early stages of the design cycle. It is anticipated that more and more hybrid tests will be conducted in aero industry like already happening in some other industries, such as civil and ground vehicle industries [10].

\section{CONCLUSION}

It is a clear trend that more and more advanced virtual testing and hybrid simulation methods are going to be used in evaluating airplane designs. The traditional Testing Pyramid approach that emphasis on uniaxial coupon test and full structure certification test is changing. Full scale strucutral certification testing is mandated for certification, so this testing will continue but the test will be able to run faster with MAC and the structure will be better known because of the new tools being developed. Through examples, this work demonstrated virtual testing models can be used to accurately predict the performance of physical test systems. Virtual testing systems can be used to setup physical tests, obtain tuning parameters, and rehearse the tests.

Hybrid simulation enables testing only part of the structure and model the rest. As a result, both analysis model and physical test can potentially be simplified significantly. Since hybrid simulation does not require all components to be tested physically, it can be conducted at a much earlier development stage.

\section{REFERENCES}

[1] Barton J., Thomsen O., (2017) Towards a New Paradigm for High Fedility Testing and Integrated Multi-Scale Modeling of Substructures and Components, 2nd International Symposium on Multiscale Experimental Mechanics (ISMEM 2017) Symposium.

[2] Laustsen, S., Lund, E., Kuhlmeier, L. and Thomsen, O.T. (2014) Failure behaviour of grid-scored foam cored composite sandwich panels for wind turbine blades 
subjected to realistic multiaxial loading conditions, Journal of Sandwich Structures and Materials, 16, (5), pp. 481-510.

[3] Ostergaard M., Ibbotson A., Roux O., Prior A., (2011) Virtual testing of aircraft structures, CEAS Aeronaut J (2011) 1:83-103

[4] Werner S., Goetze M., Sachse M., Stankovic Z., and Lance Howes L. (2015) Comparison of numerical and experimental results for the door surround structure of a pressurized fuselage, 29th ICAF Symposium - Nagoya.

[5] Wallace M.I., Wagg D.J., Neild S.A., Bunniss P., Lieven N.A.J., Crewe A.J. (2007) Testing coupled rotor blade-lag damper vibration using real-time dynamic substructuring, Journal of Sound and Vibration 307 (2007) 737-754

[6] McKenna F., Fenves G.L., and Scott, M.H. (2000). Open System for Earthquake Engineering Simulation, Univ. of California, Berkeley, California.

[7] Chen C, Ricles JP, Entwistle J (2009): Analysis of actuator delay compensation methods for real-time testing. Engineering Structures, 31, 2643-2655.

[8] Schellenberg AH, et al. (2006), Application of an Experimental Software Framework to Hybrid Simulation of Structures Through Collapse, First European Conference on Earthquake Engineering and Seismology, Paper Number: 1221, Geneva, Switzerland.

[9] Schellenberg AH, et al. (2015), Hybrid Simulation of Seismic Isolation Systems Applied to an APR-1400 Nuclear Power Plant. Pacific Earthquake Engineering Research Center, Report No. PEER 2015/05, Berkeley, CA, USA.

[10] You S, Fricke D. (2011), Advances of Virtual Testing and Hybrid Simulation in Automotive Performance and Durability Evaluation, SAE Paper No. 11M-0297. 